\title{
Use of High Pressure NMR Spectroscopy to Rapidly Identify Proteins with Internal Ligand-Binding Voids
}

\author{
Donald Gagné ${ }^{1, \dagger}$, Roksana Azad ${ }^{1,2}$, Uthama R. Edupuganti ${ }^{1,2}$, Justin Williams ${ }^{1, \ddagger}$, \\ James M. Aramini ${ }^{1}$, Kazuyuki Akasaka ${ }^{3}$, Kevin H. Gardner ${ }^{1,4,5, *}$
}

${ }^{1}$ Structural Biology Initiative, CUNY Advanced Science Research Center, New York, NY 10031, USA

${ }^{2}$ Ph.D. Program in Biochemistry, Graduate Center, City University of New York, NY 10016, USA

${ }^{3}$ Kyoto Prefectural University of Medicine, Kyoto 602-8566, Japan

${ }^{4}$ Department of Chemistry and Biochemistry, City College of New York, NY 10031, USA

${ }^{5} \mathrm{Ph} . \mathrm{D}$. Programs in Biochemistry, Chemistry and Biology Ph.D. Programs, Graduate

Center, City University of New York, NY 10016, USA

†: current address: KisoJi Biotechnology, Montréal, QC, Canada

‡: current address: Department of Physiology and Cellular Biophysics, Columbia

University Irving Medical Center, New York, NY 10032

\section{*: address correspondence to:}

Kevin Gardner, Structural Biology Initiative, CUNY Advanced Science Research Center, 85 St. Nicholas Terrace, New York, NY 10031 USA, E-mail: kgardner@gc.cuny.edu; phone: +1 2124133220

Author ORCIDs:

Donald Gagné: 0000-0002-3938-6567

Roksana Azad: 0000-0001-9909-2889

Uthama R. Edupuganti: 0000-0003-0571-4101

Justin Williams: 0000-0003-4108-5808

James M. Aramini: 0000-0001-8926-4210

Kazuyuki Akasaka: 0000-0002-9611-9086

Kevin H. Gardner: 0000-0002-8671-2556

Classification: Biological Sciences / Biophysics and Computational Biology

Keywords: high pressure NMR, ligand binding, protein cavities 


\section{Abstract}

2 Small molecule binding within internal cavities provides a way to control protein function

3 and structure, as exhibited in numerous natural and artificial settings. Unfortunately,

4 most ways to identify suitable cavities require high-resolution structures a priori and may

5 miss potential cryptic sites. Here we address this limitation via high-pressure solution

6 NMR spectroscopy, taking advantage of the distinctive nonlinear pressure-induced

7 chemical shift changes observed in proteins containing internal cavities and voids. We

8 developed a method to rapidly characterize such nonlinearity among backbone ${ }^{1} \mathrm{H}$ and

$9{ }^{15} \mathrm{~N}$ amide signals without needing to have sequence-specific chemical shift

10 assignments, taking advantage of routinely available ${ }^{15} \mathrm{~N}$-labeled samples,

11 instrumentation, and 2D ${ }^{1} \mathrm{H} /{ }^{15} \mathrm{~N}$ HSQC experiments. From such data, we find a strong

12 correlation in the site-to-site variability in such nonlinearity with the total void volume

13 within proteins, providing insights useful for prioritizing domains for ligand binding and

14 indicating mode-of-action among such protein/ligand systems. We suggest that this

15 approach provides a rapid and useful way to rapidly assess otherwise hidden dynamic

16 architectures of protein that reflect fundamental properties associated with ligand

17 binding and control. 


\section{Significance Statement}

20 Many proteins can be regulated by internally binding small molecule ligands, but

21 it is often not clear a priori which proteins are controllable in such a way. Here we

22 describe a rapid method to address this challenge, using solution NMR spectroscopy to

23 monitor the response of proteins to the application of high pressure. While the locations

24 of NMR signals from most proteins respond to high pressure with linear chemical shift

25 changes, proteins containing internal cavities that can bind small molecule ligands

26 respond with easily identified non-linear changes. We demonstrate this approach on

27 several proteins and protein/ligand complexes, suggesting that it has general utility. 


\section{Introduction}

Small molecule cofactors and ligands play critical roles in controlling protein structure and function, the understanding of which often gives insight both into natural

31 and artificial modes of regulation. Of particular interest are identifying sites where the

32 irregular structures of proteins give rise to cavities, voids and other features which can

33 serve as internal sites for such compounds to bind (Fig. 1) and allosterically control

34 protein structure and function (1,6-9). The traditional method to identify such locations

35 - high resolution X-ray crystallography, ideally with better than $2 \AA$ resolution to aid the 36 identification of internally bound waters - can be powerful, particularly when combined

37 with computational analyses for cavity identification $(5,10-16)$ or experimentally solving

38 multiple structures of proteins soaked with different organic solvents or small molecule

39 fragments $(17,18)$. Accordingly, this approach relies on having well-diffracting crystals,

40 which are not always available for all systems and can be time-consuming to produce

41 even when successful.

As an alternative method to experimentally determine which proteins might

43 contain internal cavities suitable for ligand binding, we explored the potential for using

44 high pressure solution NMR to do so. We thought this approach might be useful given

45 the need for proteins to undergo dynamic changes to allow for ligand binding within pre-

46 existing internal cavities and voids (Fig. 1). While such changes may occur rarely at

47 ambient pressure, elevated pressure will easily increase the equilibrium populations of

48 the less populated, low-volume conformers associated with hydration of cavities and

49 voids (20). Such low-lying excited state conformers (N'), if present, will usually

50 equilibrate with the ground state folded conformer $(\mathrm{N})$ rapidly on the NMR time scale 


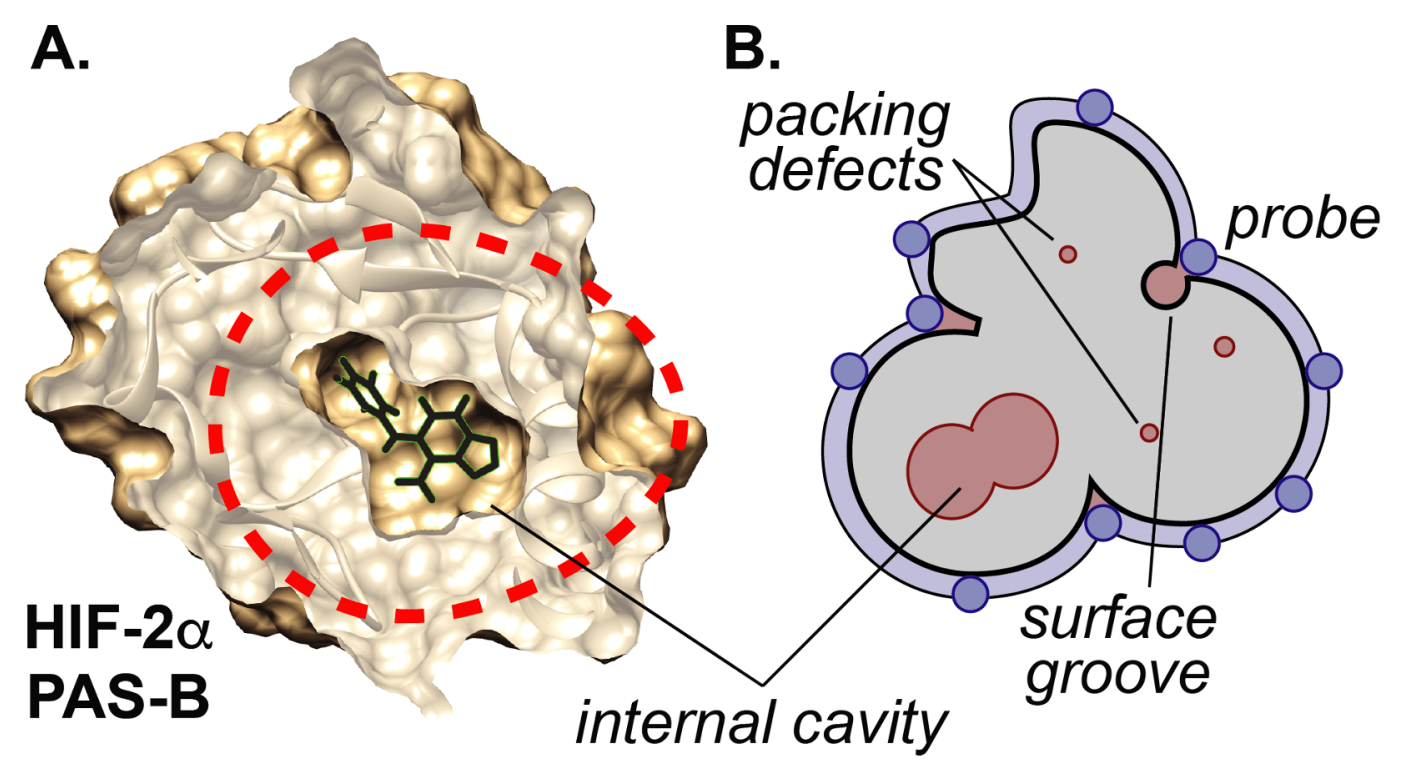

Fig. 1: Example of internal ligand binding cavity and computational analysis. $A$. Example of protein/ligand complex utilizing an internal cavity (HIF-2 $\alpha$ PAS-B, PDB: 3f1o (6)) that is sequestered over $6 \AA$ from solvent. In the apo- form of this protein (not shown), a preformed $290 \AA^{3}$ cavity with 8 crystallographically-ordered water molecules is present at this site. B. Schematic definitions of cavities, voids, and algorithm used by Protein Volume (5) as used for Fig. 3 and onwards in the manuscript. We refer to cavities as internal openings larger than a single water molecule $\left(V>30 \AA^{3}(19)\right)$, while voids are more generally non-protein filled spaces that include cavities along with other types of packing defects distributed throughout and around a protein. With a protein structure available (gray), the total void volume can be straightforwardly calculated by the difference of the solvent-excluded volume of a protein (generated by rolling probes over the molecular surface, typically with water-sized radii) and the volume taken by protein atoms = volume under blue surface minus gray volume (5).

51 ( $\tau<<m s)$, giving rise to averaged single peaks in multidimensional spectra which exhibit

52 non-linear chemical shift changes as pressure affects the $\mathrm{N} \rightleftharpoons \mathrm{N}^{\prime}$ equilibrium (21).

53 Based on prior work by several groups (21-24), we anticipated that this effect might be

54 easily detected by solution NMR at pressures in the 1000-2000 bar range, below the

55 pressures which typically partially or completely unfold proteins $(21,25,26)$.

To evaluate this approach, we examined the effects of high hydrostatic pressure

57 on the NMR chemical shifts of a collection of protein domains and protein/ligand 
complexes. Many of these proteins are members of the Per-ARNT-Sim (PAS) family of ligand-controlled protein/protein interaction domains, which often internally bind different small molecule cofactors to sensitize them to environmental factors like $\mathrm{O}_{2}$, light, and

61 xenobiotics $(27,28)$. Changes to the occupancies or configurations of these cofactors

62 trigger conformational changes in the surrounding protein, regulating the activity of

63 various effector domains in natural and engineered proteins $(28,29)$. While a number

64 of high-resolution structures of apo- and ligand-bound PAS domains have been solved,

65 these models and predictions which can be made from them can provide insights into

66 only a small fraction of the many thousands of "orphan" PAS domains without known

67 ligands. Complementing these proteins, we added additional proteins and

68 protein/ligand complexes from a wide range of domain types - including some which

69 are known to bind ligands internally, some not - to establish the generality of this

70 approach for quickly probing protein structure and function.

$71 \quad$ Here we test the ability of high-pressure NMR to rapidly identify void-containing

72 proteins, with three key advances. First, by analyzing pressure titration data from over

7340 proteins and protein/ligand complexes, we show that an easily accessible metric -

74 how differently sites within a protein respond to increasing pressure, as assessed by the

75 diversity of non-linear chemical shift perturbations observed in a simple titration without

76 requiring site-specific assignments - correlates well with the void volume within a

77 protein. We find that this metric is robust enough to predict total void volume on its own,

78 allowing the prioritization of potential ligand-binding capability amongst several targets.

79 Second, we demonstrate that internal ligand binding within a protein can reduce this

80 heterogeneity, quickly providing information on ligand binding and, in certain cases, 
81 mode of action. Finally, we illustrate how this method can also be used to rapidly

82 assess the impact of point mutations/repacking, such as those used to fill or generate

83 cavities to facilitate artificial control, on the prevention or enabling of water entry. Taken

84 together, our data show the general utility of these rapidly acquired, easily analyzed

85 data to provide this important biophysical characterization of new proteins.

\section{Results}

88 Evaluation of non-linear NMR chemical shift responses to high pressure: We began by

89 examining the pressure dependence of NMR signals from a set of well-characterized

90 proteins using the workflow in Fig. 2. For each $\mathrm{U}^{15} \mathrm{~N}$ labeled protein sample, we

91 acquired ${ }^{1} \mathrm{H} /{ }^{15} \mathrm{~N} \mathrm{HSQC}$ spectra at increasingly higher pressures from 20-2500 bar. After

92 each individual dataset was acquired at high pressure, we lowered the pressure to 20

93 bar and acquired a spectrum to confirm the reversibility of conformational changes; after

94 any sign of irreversible changes in peak intensity or location, the pressure series was

95 stopped. These series were typically composed of 21 spectra each taking $60 \mathrm{~min}$

96 apiece, for a total of approximately $21 \mathrm{hr}$. Post-processing, peaks were picked and the

97 pressure dependence of changes of their ${ }^{1} \mathrm{H}$ and ${ }^{15} \mathrm{~N}$ chemical shifts were

98 independently fit to a second-order polynomial equation:

99

$$
\delta_{i}=a_{i}+b_{i} p+c_{i} p^{2} \text { (Eq. 1) }
$$



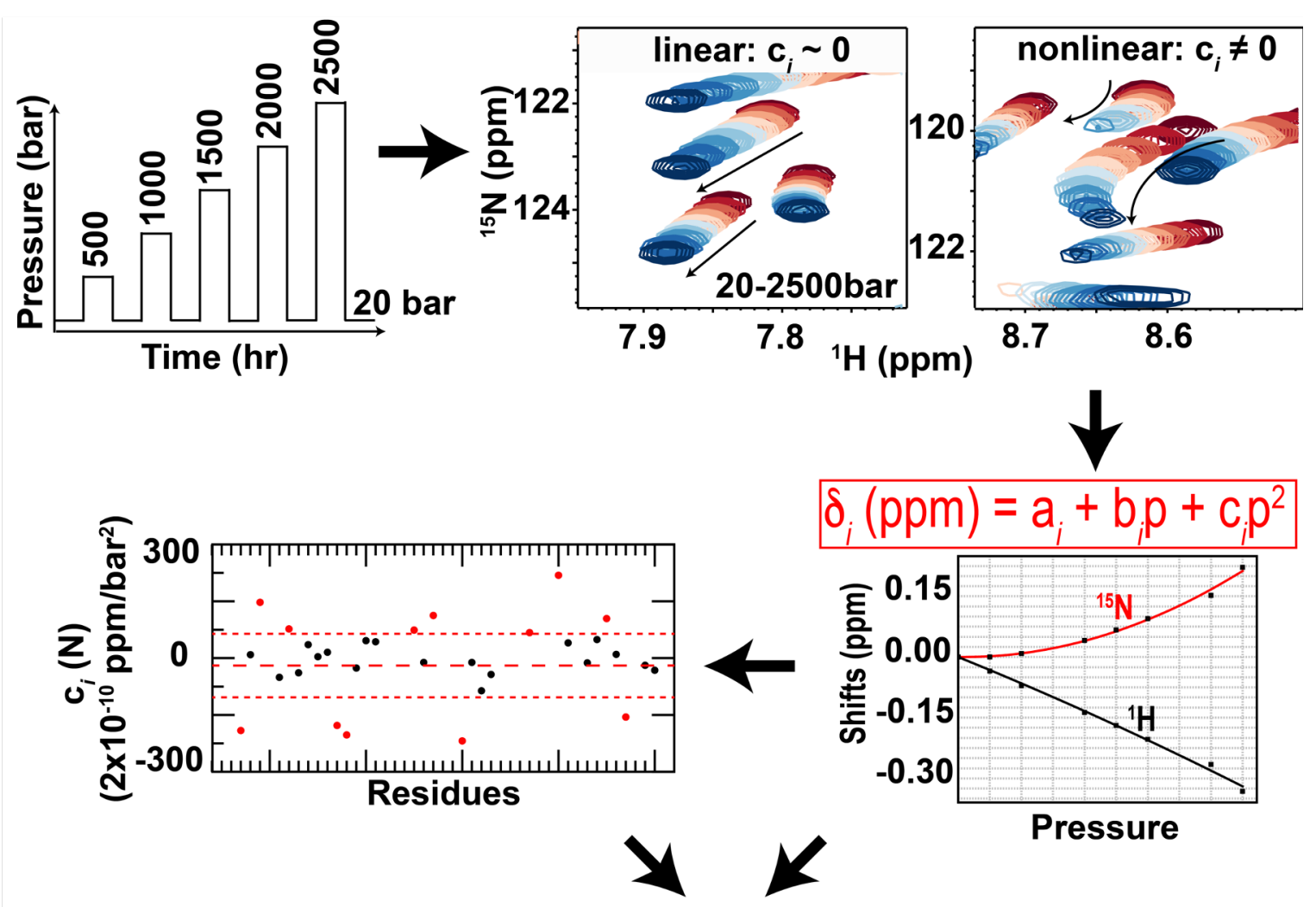

Pressure

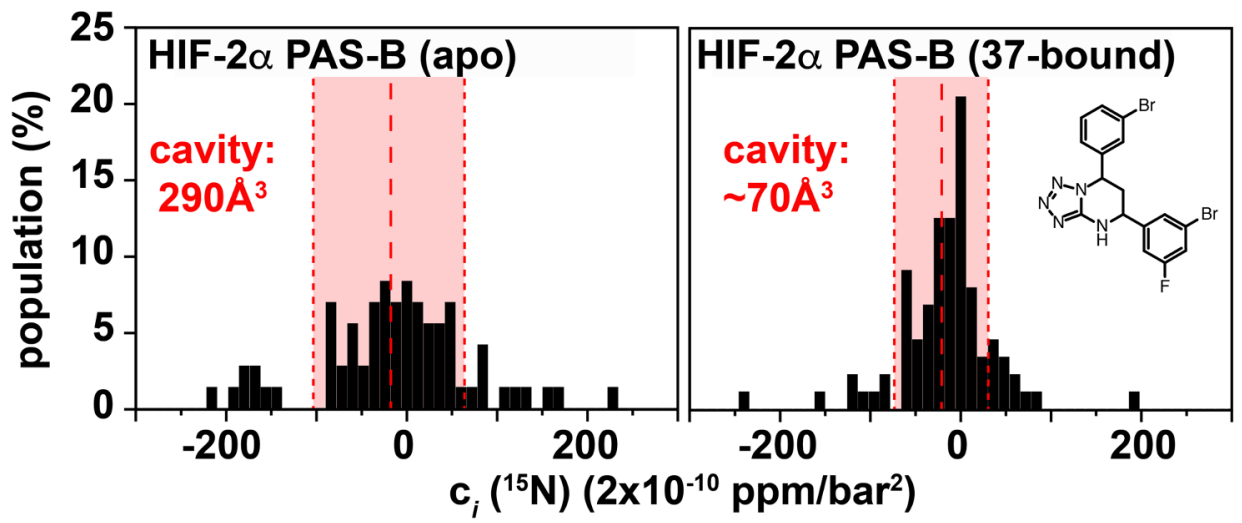

Fig. 2: Workflow of pressure NMR analyses. For each protein analyzed, ${ }^{1} \mathrm{H} /{ }^{15} \mathrm{~N} H S Q C$ spectra were acquired at increasing pressures from 20-2500 bar, interleaving additional spectra at 20 bar between those at higher pressure to assess protein reversibility. Following processing, peaks were picked and chemical shifts monitored as a function of increasing pressure. Independently treating ${ }^{1} \mathrm{H}$ and ${ }^{15} \mathrm{~N}$ movements, the pressure-dependent chemical shift changes of each peak are fit to the second order polynomial indicated to obtain linear $\left(b_{i}\right)$ and nonlinear $\left(c_{i}\right)$ coefficients. Subsequent analyses of the nonlinear $\left(c_{i}\right)$ coefficients include either plotting $c_{i}$ values as a function of residue number (for proteins with backbone chemical shift assignments) to identify regions with likely pressure-dependent conformational changes or to simply generate histograms of $c_{i}$ values to give a quick initial characterization of likely ability to adopt multiple folded conformations. 
As established by Akasaka and co-workers $(21,25)$, the linear $\left(b_{i}\right)$ and nonlinear

$\left(c_{i}\right)$ coefficients of these pressure responses reflect different properties of each protein.

To evaluate various analyses of these data, we examined an initial group of nine

106 proteins ("test set") with high resolution structures with total void volumes ranging from

107 approximately $1500-8500 \AA^{3}$ as assessed by ProteinVolume (5). This approach

108 provides a sum of the volumes of a wide array of packing defects, cavities, etc.

109 regardless of their size or distribution within a protein structure by simply calculating the

110 difference between the solvent-accessible volume of a protein and the volume occupied

111 by protein atoms (Fig. 1B). Pressure titration data were additionally recorded from

112 more than 30 additional proteins and protein ligand complexes, with varying degrees of

113 structural information to build the "complete set" of data for subsequent analyses.

As an initial analysis, we examined the absolute values of the two chemical shift

115 coefficients $\left(\left|b_{i}\right|,\left|c_{i}\right|\right)$, separately averaged over all backbone amide protons and

116 nitrogens (typically 25-125) within each protein. From these analyses, we confirmed

117 that the averaged ${ }^{1} \mathrm{H}$ and ${ }^{15} \mathrm{~N}\left|\mathrm{~b}_{i}\right|$ values were fairly uniform across proteins with less

118 than two-fold variation, while the corresponding $\left|c_{i}\right|$ values varied over $4-10$ fold ranges

119 (Fig. S1). These data expand the original data sets of Akasaka and Li (25) from seven

120 proteins and complexes to over 40 total, add an independent measurement and

121 analysis of the GB1 protein to assess the impact of different equipment, labs, and

122 software for data acquisition and analyses (e.g. $750 \mathrm{MHz}$ in Kobe University by K.A.;

$123700-800 \mathrm{MHz}$ in New York by K.H.G.), and support the original interpretations that the

124 linear $\left|b_{i}\right|$ component is relatively fixed among systems while $\left|c_{i}\right|$ depends on a protein-

125 specific feature (25). 
Further investigating these pressure-dependent chemical shift changes, we noted a trend towards proteins with larger total void volumes having both higher $\mathrm{c}_{i}$ values (Fig. S1) and a greater range of individual residue-specific $c_{i}$ parameters than

129 proteins with smaller voids, both of which we thought might reflect a more heterogenous structural response. While the larger $c_{i}$ values are captured by the previously-described 131 average $\left|c_{i}\right|$ parameter (25), we examined several ways to quantitate the heterogeneity 132 in $c_{i}$ values, settling on a combination of histograms and the standard deviation of the $c_{i}$ $133\left(\operatorname{stdev}\left(c_{i}\right)\right)$. While the correlation between average $\left|c_{i}\right|$ and $\operatorname{stdev}\left(c_{i}\right)$ values for amide ${ }^{1} H$ 134 and ${ }^{15} \mathrm{~N}$ shifts (Fig. S2) suggest similarities between the two metrics, we opted to 135 proceed using $\operatorname{stdev}\left(c_{i}\right)$ to take advantage of the larger range of values provided by 136 dropping the absolute value operation. We also observed a high correlation between 137 the $\operatorname{stdev}\left(\mathrm{c}_{i}\right)$ values of ${ }^{1} \mathrm{H}$ and ${ }^{15} \mathrm{~N}$ (Fig. S3), suggesting that non-linear shift changes of 138 either nucleus report on pressure-induced changes despite differences in the structural 139 factors which influence them (30). Our subsequent analyses utilized $\operatorname{stdev}\left(\mathrm{c}_{i}\right)$ of ${ }^{15} \mathrm{~N}$ 140 chemical shift changes $\left(=\operatorname{stdev}\left(\mathrm{c}_{i}\left[{ }^{15} \mathrm{~N}\right]\right)\right)$, which are thought to be most strongly 141 influenced by changes in backbone torsion angles $(25,30)$. To examine the linkage of $\operatorname{stdev}\left(c_{i}\right)$ to total void volume, we measured the

143 nonlinear components $\left(\mathrm{C}_{i}\left[{ }^{15} \mathrm{~N}\right]\right)$ of a test set of nine proteins (with one, GB1, repeated in 144 duplicate) with known structures and a range of total void volumes (Fig. 3). Histograms 145 of $\operatorname{stdev}\left(\mathrm{c}_{i}\right)$ parameters exhibited the previously-mentioned variability, with proteins 146 having larger total void volumes typically having broader distributions than those with 147 smaller total void volumes (Fig 3 inset). We interpreted this trend as stemming from 148 voids enabling proteins to increasingly shift from the native $\mathrm{N}$ conformation to a second 


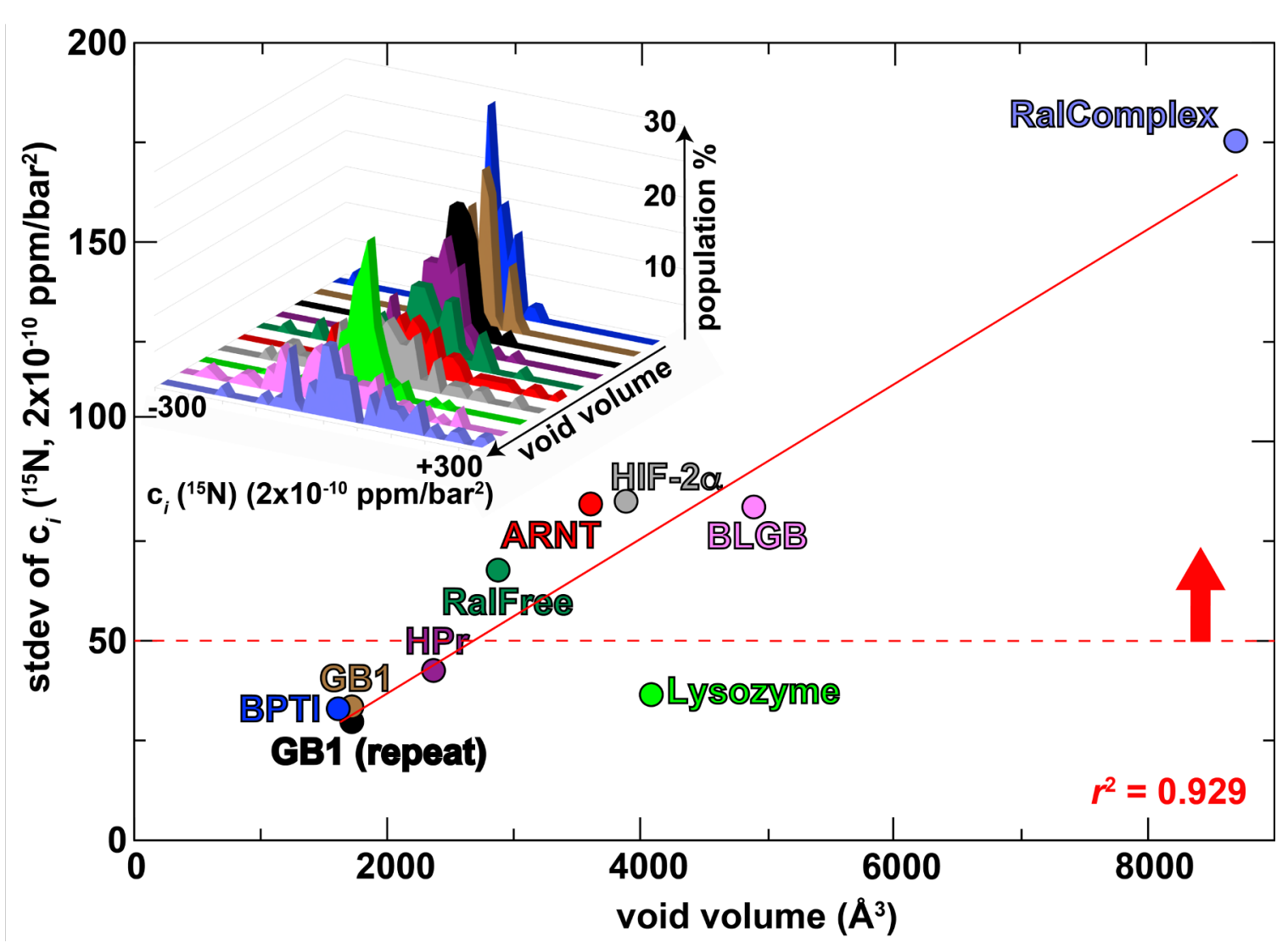

Fig. 3: Increasing diversity of non-linear pressure dependent chemical shift changes correlates with increased void volume. Total void volumes calculated by ProteinVolume (5) for each proteins are presented on the $x$-axis; the $y$-axis plots the standard deviation of the non-linear $\mathrm{c}_{i}$ parameters for the amide ${ }^{15} \mathrm{~N}$ chemical shifts of each protein, typically using data from 25-125 crosspeaks for each protein. The linear regression (red line, $y=-1.564+0.0194 x$, omitting the lysozyme point) shows a correlation coefficient $\left(r^{2}\right)$ of 0.929 . The red dashed line indicates an arbitrary value of approximately $50\left(\times 2 \times 10^{-10}\right) \mathrm{ppm} / \mathrm{bar}^{2}$ as suggesting the potential for small molecule binding, as noted in the text. Inset: histograms of each of the ten proteins showing the distributions of measured ${ }^{15} \mathrm{~N} \mathrm{c}_{i}$ parameters for the same set of proteins (as indicated by the same color scheme).

149 folded N' conformation under pressure, reflected in the non-linear chemical shift

150 responses as the N' is progressively populated. Quantitating the breadth of these

151 distributions by $\operatorname{stdev}\left(\mathrm{c}_{i}\left[{ }^{15} \mathrm{~N}\right]\right)$ and plotting these versus total void volume showed a

152 linear correlation coefficient $\left(r^{2}\right)$ of 0.929 , supporting the linkage between pressure-

153 induced chemical shift nonlinearities to identify cavities. Of note, the proteins with a

$154 \operatorname{stdev}\left(\mathrm{c}_{i}\left[{ }^{15} \mathrm{~N}\right]\right)$ above $50\left(\times 2 \times 10^{-10} \mathrm{ppm} / \mathrm{bar}^{2}\right)$ are all known to bind other proteins or 
small molecules, suggesting an arbitrary value which could be useful to prioritize for ligand-screening.

We note that the primary outlier of the observed linear correlation, hen egg white

158 lysozyme, is quite thermostable with a $T_{m}$ of almost $75^{\circ} \mathrm{C}(31)$, likely hampering its

transition into an excited state under pressure unless its intrinsic stability of its basic

160 folded state is substantially lowered (e.g. by cooling close to the cold denaturation

161 temperature (32)). In addition, we cannot rule out potential contributions from lysozyme

162 being an enzyme instead of a signal transduction component, which may contribute a

163 different ability to adopt alternative conformations reflected in pressure-induced non-

164 linear chemical shift changes. This remains to be seen in future studies.

Use of pressure NMR to reveal ligand binding mode of action - Well-defined cases:

167 Our correlation between increased heterogeneity of pressure-induced chemical shift changes and void size makes a strong prediction that this route should provide a rapid way to assess ligand binding: ligands which bind within the protein and reduce total void

170 volume should decrease the $\operatorname{stdev}\left(c_{i}\right)$ value of spectra recorded on the receptor. To test

171 this prediction, we evaluated how ligand binding affects the PAS-B domains of the

172 human HIF-2 $\alpha$ and ARNT proteins, both of which are involved in the human hypoxia

173 response (33) and contain internal cavities known to bind artificial small molecule

174 ligands $(1,3,6)$.

176 have shown that it contains a single internal water-filled $290 \AA^{3}$ cavity with no obvious

177 access to external solvent. However, a variety of screening efforts - from NMR-based 
178 fragment binding screens to high throughput screens of HIF-2 $\alpha /$ ARNT disruption (1, 2,

$1796,35)$ or protein stability (35) - have identified a wide range of small molecules which

180 bind into this cavity with nano- to micromolar affinities. High-resolution X-ray structures

181 of these complexes show that the ligands displace water and reduce HIF-2 activity by

182 impacting HIF-2 $\alpha /$ ARNT interactions (36-38). To accommodate these binding events

183 within a solvent-inaccessible cavity, the HIF-2 $\alpha$ PAS-B protein must dynamically

184 fluctuate to allow for a conformation which allows the entry of small molecules into the

185 interior (39). Our pressure NMR analysis confirmed this hypothesis, showing a very

186 broad distribution of $\mathrm{c}_{i}\left({ }^{15} \mathrm{~N}\right)$ responses with a correspondingly large $\operatorname{stdev}\left(\mathrm{c}_{i}\left[{ }^{15} \mathrm{~N}\right]\right)$ of 85

$187 \times\left(2 \times 10^{-10} \mathrm{ppm} / \mathrm{bar}^{2}\right)$ (Figs. 4A and S4). We anticipated that repeating these

188 measurements in the presence of two nanomolar-affinity compounds (2 (1) and 37 (2))

189 would show smaller non-linear chemical shift changes than the apo protein given the

190 smaller total void volume and expected reduced flexibility of the protein/ligand

191 complexes. Our data supported this, as we observed decreases in $\operatorname{stdev}\left(\mathrm{c}_{i}\left[{ }^{[5} \mathrm{N}\right]\right)$ from

$19285 \times\left(2 \times 10^{-10} \mathrm{ppm} / \mathrm{bar}^{2}\right)$ for the apo protein to 64 (2-bound) and 53 (37-bound) $\times\left(2 \times 10^{-10}\right.$

$\left.193 \mathrm{ppm} / \mathrm{bar}^{2}\right)$, respectively. Notably, these decreases do not solely correlate with loss of

194 void volume in some cases: measurements conducted on HIF-2 $\alpha$ D1, a

195 computationally-repacked variant with five point mutations which reduce the cavity

196 volume to $77 \AA^{3}(40)$ while retaining function, shows an increase in $\operatorname{stdev}\left(\mathrm{c}_{i}\left[{ }^{15} \mathrm{~N}\right]\right)$ up to 

and natural proteins contribute to non-linear responses to pressure.
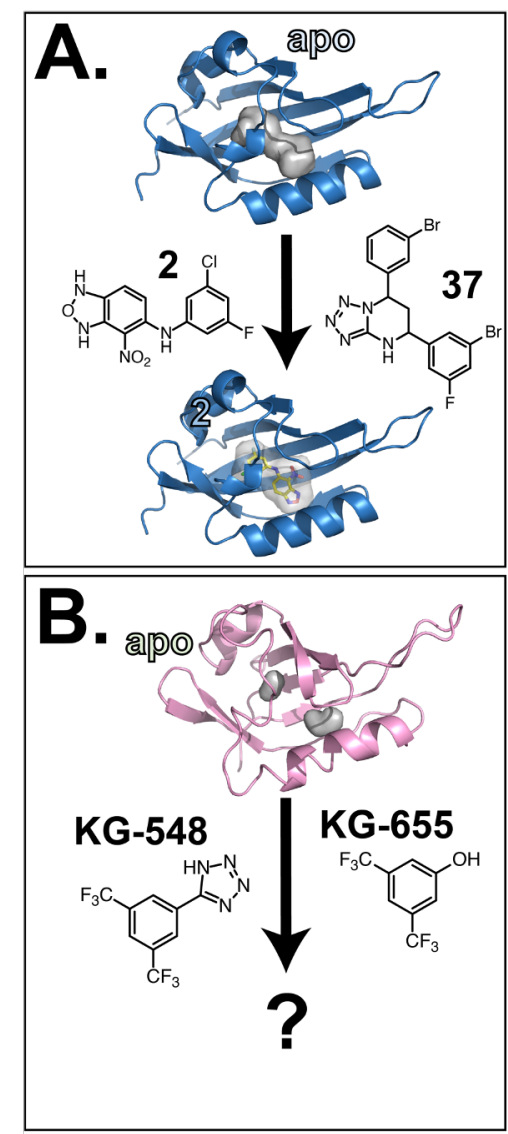
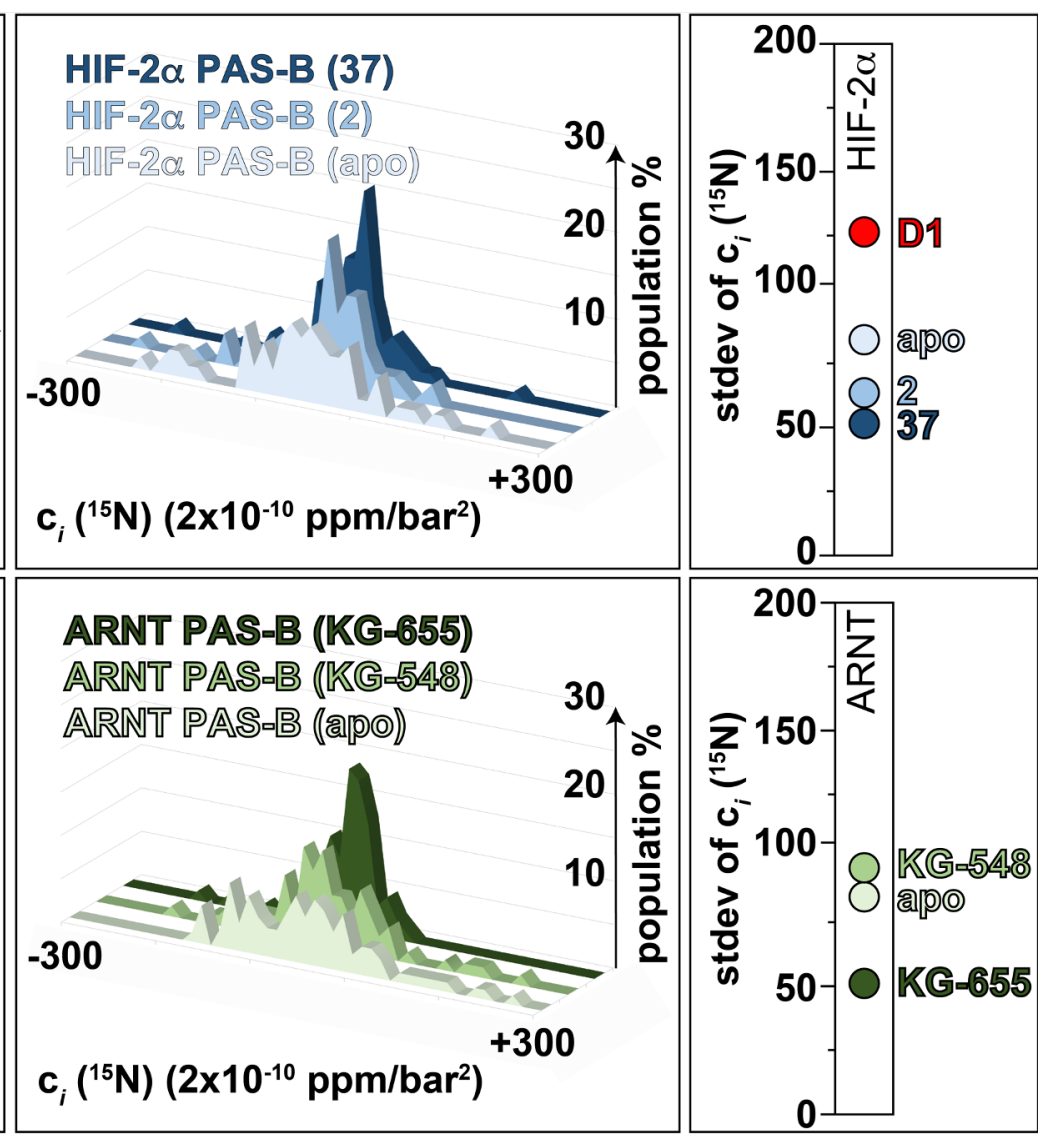

Fig. 4: High pressure NMR can provide mode-of-action information on ligand binding, even in the absence of structural information. (Left panel) $A$. Three-dimensional ribbon diagrams of HIF-2 $\alpha$ PAS-B in its apo (PDB: $3 f 1 p$ ) and holo forms (complexed with 2, PDB: 4ghi), highlighting the location of an internal cavity for ligand binding. Chemical structures of two high-affinity inhibitors, 2 and $\mathbf{3 7}(1,2)$ are also shown. B. Three-dimensional ribbon diagram of ARNT PAS-B (PDB: 3f1p) highlighting the location of an internal cavities, along with the structures of two moderate affinity binders, KG-548 and KG-655 (3). No structural details are available for the bound form. (Central panel) Histograms of the ${ }^{15} \mathrm{~N} \mathrm{c}_{i}$ coefficients measured on apo- and holo forms of HIF-2 $\alpha$ and ARNT PAS-B domains using the approach diagrammed in Fig. 2. (Right panel) Comparisons of the $\operatorname{stdev}\left(\mathrm{c}_{i}\left[{ }^{15} \mathrm{~N}\right]\right)$ values of $H I F-2 \alpha$ and ARNT PAS-B apo and ligand-bound samples, showing substantial effects as cavities are occupied by water (apo forms), small molecule ligands or protein sidechains. 
We used the same approach with the ARNT PAS-B domain, which has the same tertiary structure as HIF-2 $\alpha$ PAS-B but contains smaller internal cavities which total 150

\section{2} $\AA^{3}$ in volume. We have previously used NMR-based fragment screening, isothermal titration calorimetry (ITC) and microscale thermophoresis (MST) to identify several compounds which bind ARNT PAS-B with micromolar affinities; two of these, KG-548 and KG-655, are further known to disrupt ARNT PAS-B interactions with coactivator proteins (3). However, the binding modes of these compounds remain unclear without

207 co-complex structures solved by NMR or X-ray crystallography. By acquiring pressure

208 NMR data, we confirmed that ARNT PAS-B retains a flexibly-accessible interior cavity 209 with a stdev $\left(\mathrm{c}_{i}\left[{ }^{15} \mathrm{~N}\right]\right)$ of $84 \times\left(2 \times 10^{-10} \mathrm{ppm} / \mathrm{bar}^{2}\right)$ (Figs. 4B and S5). Repeating these in

210 the presence of the KG-655 ligand, we observed a drop of $\operatorname{stdev}\left(\mathrm{c}_{i}\left[{ }^{15} \mathrm{~N}\right]\right)$ to $51 \times\left(2 \times 10^{-10}\right.$

$\left.211 \mathrm{ppm} / \mathrm{bar}^{2}\right)$, consistent with an interior binding location for this small ligand. However,

212 similar studies with the larger KG-548 ligand show a $\operatorname{stdev}\left(\mathrm{c}_{i}\left[{ }^{15} \mathrm{~N}\right]\right)$ comparable to the 213 apo protein, with an observed value of $96 \times\left(2 \times 10^{-10} \mathrm{ppm} / \mathrm{bar}^{2}\right)$ that suggest that this

214 compound binds in a different mode than KG-655, potentially outside the cavity.

217 demonstration of this approach, we examined its utility for PAS domains outside the 218 hypoxia response and with less well understood regulation. We considered two such 219 targets, the first of which is the N-terminal PAS domain of human PAS kinase (PASK 220 PAS-A), a PAS domain-regulated serine/threonine kinase conserved among eukaryotes $221(4,41)$. This domain has a canonical PAS structure, with a five-stranded $\beta$-sheet 222 flanked to one side by several $\alpha$ helices $(27,42)$. While only a small surface groove of 
$22373 \AA^{3}$ could be identified near the F/G loop in the representative member of the solution

224 structure ensemble, an NMR-based fragment screen of 750 compounds identified

225 several small molecules that bound with micromolar affinity to PASK PAS-A (4).

226 Chemical shift perturbations suggest that these compounds bind within the domain

227 interior, analogous to the subsequently-discovered HIF-2 $\alpha$ and ARNT PAS-B binding

228 compounds, but the lack of a PASK PAS-A/ligand complex structure leaves this as an

229 open issue. To address this, we used high-pressure NMR to examine the response of

230 PASK PAS-A in its apo form and when saturated with two compounds with $K_{D}=13-24$

$231 \mu \mathrm{M}$ affinities (KG-535, KG-571 = compounds 1 and 2 in (4)). For the apo- protein, we

232 observed a stdev $\left(\mathrm{c}_{i}\left[{ }^{15} \mathrm{~N}\right]\right)$ of $45 \times\left(2 \times 10^{-10} \mathrm{ppm} / \mathrm{bar}^{2}\right)$; from the correlation identified in

233 our test set (Fig. 3), this value predicts that PASK PAS-A contains a total void volume

234 slightly smaller than the $3345 \AA^{3}$ calculated from the representative member of the

235 solution structure ensemble (Figs. 5A and S6). Upon addition of the KG-535 and KG-

236571 ligands, we observed smaller stdev $\left(\mathrm{c}_{i}\left[{ }^{15} \mathrm{~N}\right]\right)$ values for both ligand-bound forms (35

237 and $28 \times\left(2 \times 10^{-10} \mathrm{ppm} / \mathrm{bar}^{2}\right)$ for the KG-535 and KG-571-bound forms, respectively; Fig.

238 5A). These data suggest that both compounds bind within the void volume of PASK

239 PAS-A, with KG-571 having a greater effect on the domain adopting alternative folded

240 conformations.

We completed our analyses of natural proteins by examining a prokaryotic PAS

242 domain from a novel histidine kinase from Rhizobium etli (43), chosen because of a).

243 homology with the light-regulated PAS-histidine kinase EL346 $(44,45)$ and b). the

244 widespread usage of small molecule ligands to control the structure and function of

245 PAS-containing proteins (including many histidine kinases (28)). While no experimental 

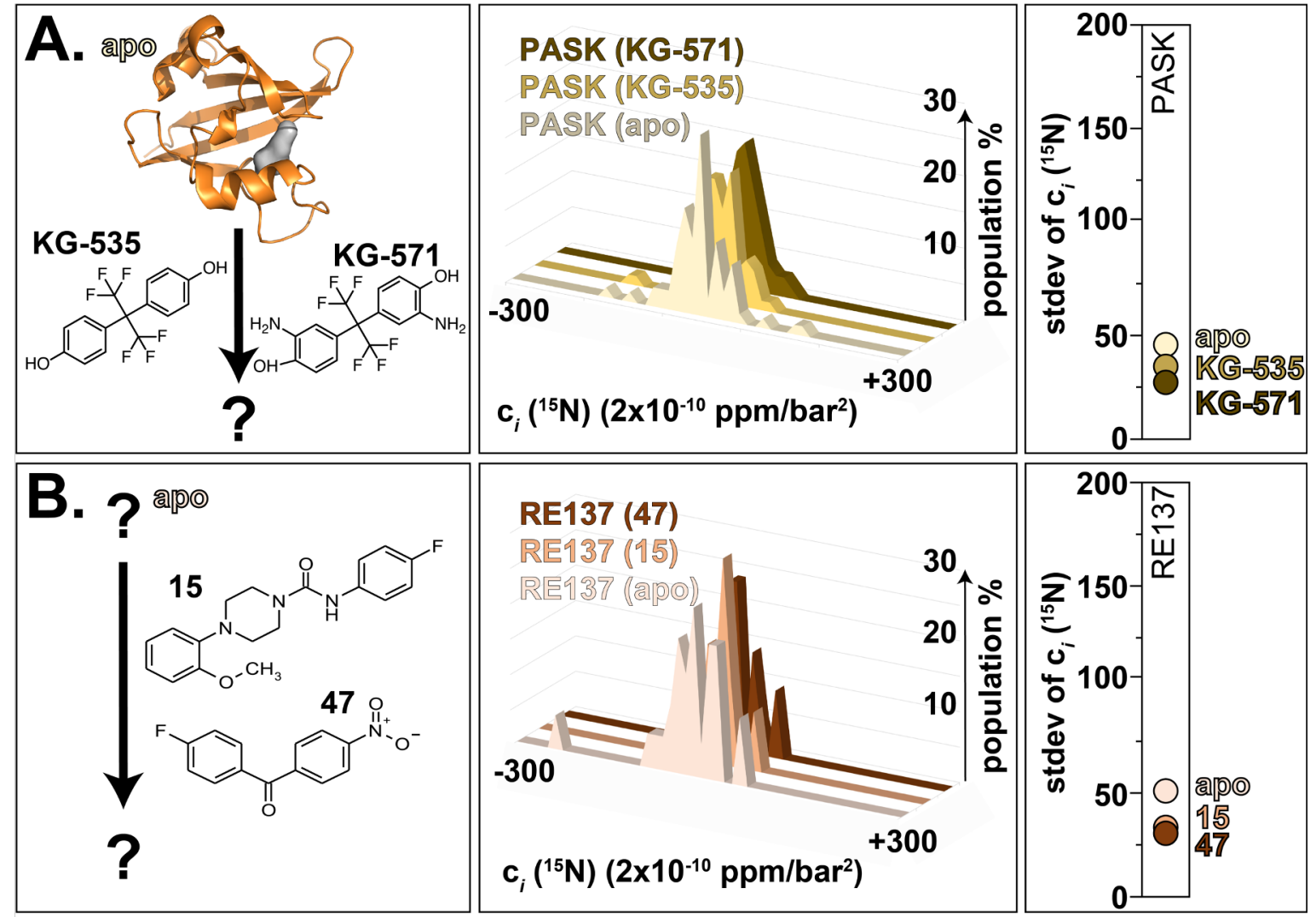

Fig. 5: High pressure NMR can provide ligand binding information, even in the absence of structural information. (Left panel) A. Three-dimensional structure of PASK PAS-A (PDB: 1II8) and structures of two binding compounds, KG-535 and KG-571 (4). No high-resolution structures of protein/ligand complexes are available. B. No structural information is available for the RE137 PAS domain, in either apo and bound forms. Structures of two binding compounds identified by ${ }^{19} \mathrm{~F}$ ligand-detected NMR screening, 15 and $\mathbf{4 7}$. (Central panel) Histograms of the ${ }^{15} \mathrm{~N} \mathrm{c}_{i}$ coefficients measured on apo- and holo forms of PASK and RE137 PAS domains using the approach diagrammed in Fig. 2. (Right panel) Comparisons of the $\operatorname{stdev}\left(\mathrm{c}_{i}\left[{ }^{15} \mathrm{~N}\right]\right)$ values of PASK and RE137 apo- and ligand-bound samples.

structures are available of this domain, which we designate RE137, we hypothesized

247 that the structure may contain a cavity that can accommodate a small molecule given

248 precedence from other PAS domains (28). Pressure NMR analyses of the apo protein

249 support this possibility, returning a $\operatorname{stdev}\left(\mathrm{c}_{i}\left[{ }^{15} \mathrm{~N}\right]\right)$ value of $53 \times\left(2 \times 10^{-10} \mathrm{ppm}^{\left.-\mathrm{bar}^{2}\right)}\right.$ (Figs.

250 5B and S7), suggesting the presence of a $2800 \AA^{3}$ total void volume from the

251 correlation seen in Fig. 3. To provide an initial assessment of small molecule binding, 
252 we used an $R_{2}$-filtered ${ }^{19} \mathrm{~F}$ NMR assay to search for binders of RE137 within a 100 -

253 member library of fluorinated compounds (46). For this study, we used two of those

254 compounds 15 and 47; pressure analyses of RE137 saturated with each compound

255 exhibited decreases of 43 and $39 \%$ with $\operatorname{stdev}\left(\mathrm{c}_{i}\left[{ }^{15} \mathrm{~N}\right]\right)$ values of 30 and $32 \times\left(2 \times 10^{-10}\right.$

$256 \mathrm{ppm} / \mathrm{bar}^{2}$ ) for the 15- and 47-bound forms, respectively. We interpret these data to

257 indicate that RE137 can bind both compounds in such a way as to reduce the total void

258 volume of the protein.

Use of pressure NMR to probe artificially-designed proteins: As a final demonstration of

261 the utility of this method, we examined its utility in investigating the flexibility of an

262 artificially-designed protein, CA01 (47). With advances in protein engineering enabling

263 the development of artificial ligand-binding biosensor proteins (48), we sought to probe

264 whether a protein such as CA01 - which contains a completely-enclosed $75 \AA^{3}$ cavity

265 within approximately $3500 \AA^{3}$ of total void volume - might show similar pressure-

266 dependent non-linear chemical shift changes as natively-evolved counterparts. From

267 our correlation in Fig. 3 we expected to see a stdev $\left(\mathrm{c}_{i}\left[{ }^{15} \mathrm{~N}\right]\right)$ value of approximately 75

$268\left(2 \times 10^{-10} \mathrm{ppm} / \mathrm{bar}^{2}\right)$ from a natural protein with void volume of this size, but we instead

269 observed a substantially smaller value of $38 \times\left(2 \times 10^{-10} \mathrm{ppm} / \mathrm{bar}^{2}\right)$ (Fig. 6). As CA01 is

270 extremely stable to thermal and chemical denaturation, requiring $5 \mathrm{M}$ guanidinium

271 hydrochloride to exhibit a complete thermal melt with a $75^{\circ} \mathrm{C} T_{\mathrm{m}}(47)$, we view this

272 observation supports our prior findings with lysozyme suggesting thermostable proteins

273 being less able to adopt alternative conformations (Fig. 3). 

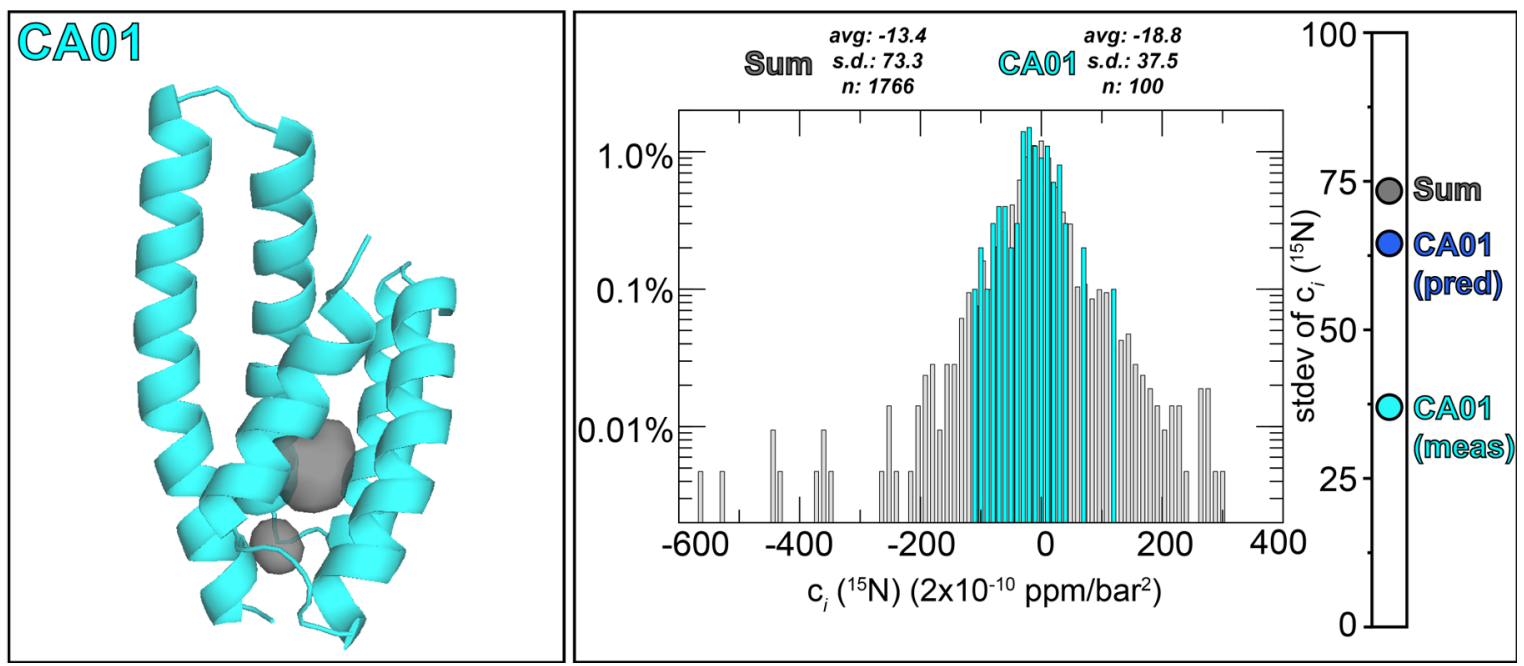

Fig. 6: High pressure NMR of a highly thermostable, cavity-containing designed protein gives smaller non-linear pressure dependent chemical shift changes than anticipated from void volume. (Left panel) Three-dimensional structure of CA01 (PDB: 5e6g), highlighting the location of a $75 \AA^{3}$ internal cavity within a total void volume of $3500 \AA^{3}$. (Right panel) Histogram of the ${ }^{15} \mathrm{~N} \mathrm{c}_{i}$ coefficients measured on CA01 (aqua) and 22 apo proteins listed in Fig. S1 (grey) using the approach diagrammed in Fig. 2. Bar on right indicates $\operatorname{stdev}\left(\mathrm{c}_{i}\left[{ }^{15} \mathrm{~N}\right]\right)$ measured for CA01 (aqua) and 22 apo proteins (grey), along with the value predicted for CA01 from the correlation in Fig 3.

\section{Discussion}

In the present work, we successfully used non-linear pressure-induced chemical

277 shift effects at backbone amide ${ }^{1} \mathrm{H}$ and ${ }^{15} \mathrm{~N}$ nuclei to rapidly assess whether proteins

278 contain substantial total void volumes within them and if these can bind small molecule

279 ligands. A particular strength of our implementation is its simplicity, as 1). the

necessary $U-{ }^{15} \mathrm{~N}$ labeled samples are routinely available from $E$. coli or in vitro

expression systems, 2). the NMR data needed are easily obtained from conventional 
Mechanistically, we attribute the non-linear chemical shift changes with

287 increasing pressure to site-specific conformational changes of the protein from the

288 native folded structural ensemble $(\mathrm{N})$ to the low-lying excited state ensemble ( $\left.\mathrm{N}^{\prime}\right)$ with

289 smaller volume (17). Our specific choice of backbone amide ${ }^{1} \mathrm{H}$ and ${ }^{15} \mathrm{~N}$ chemical shifts

290 give us complementary views of these transitions (Fig. S3), as ${ }^{1} \mathrm{H}$ chemical shifts are

291 dominated by hydrogen bonding to these atoms, while ${ }^{15} \mathrm{~N}$ chemical shifts are

292 influenced by a broader set of factors, including changes in hydrogen bonding (to the

293 both the adjacent ${ }^{1} \mathrm{H}$ and carbonyl $\mathrm{CO}$ groups), local backbone dihedrals and sidechain

294 conformations (49). Of note, non-linear pressure-dependent chemical shift changes at

295 these sites are much smaller in peptides or intrinsically disordered proteins (50).

296 Indeed, non-linear pressure-dependent amide ${ }^{1} \mathrm{H}$ and ${ }^{15} \mathrm{~N}$ chemical shift changes have

297 long been recognized as reflecting idiosyncratic differences among proteins (51). Prior

298 work by one of our groups (K.A.) suggested that this property depends on the density of

299 cavities within the protein (25); here we both expand the number of proteins examined

300 from that work and suggest that contributions likely arise from small and large voids

301 distributed throughout the protein which collectively give rise to the total void volumes

302 calculated by the program ProteinVolume (5) given the excellent correlation we present

303 in Fig. 3. We posit that such an approach reveals aspects that will be correlated to the

304 ability of proteins to be similarly affected by allosteric regulators and other ligands,

305 linking these non-linear chemical shift effects to the potential for small molecule binding 306 and regulation.

In addition to getting information on the total void volume within a given protein,

308 the strengths of this analysis include the ability to identify ligands which bind to a given 
target and (de)stabilize one conformation in a way that may provide allosteric switching. As such, this provides trivially-accessible mode-of-action information that is often

311 challenging to get otherwise without resources such as compounds of known binding

312 location for competition studies or cavity-filling point mutants. Our comparison of two

313 cavity-perturbing effects for HIF-2 $\alpha$ PAS-B - small molecule binding and cavity-filling

314 redesign - produced opposite effects, with the small molecules stabilizing the native fold

315 and mutations destabilizing in our pressure NMR analyses. We underscore that these

316 effects occur despite minor changes in ground state structure among structures of the

317 apo, ligand-bound and redesigned proteins (1, 6, 40). As such, pressure-dependent

318 chemical shift effects give us insight into details of the packing and dynamics of proteins

319 more simply than most other methods can provide. Finally, our comparison of the

ARNT PAS-B binding compounds KG-548 and KG-655 provide an outstanding example

321 of two molecules known to bind by NMR chemical shift perturbations, but with limited

322 binding site information previously available. With high pressure NMR, we predict

323 different binding modes between the two ligands, with KG-655 binding primarily to an

324 internal site while KG-548 binds externally.

We emphasize that our approach requires very little preliminary information on

326 the target, needing neither structures nor site-specific chemical shift assignments, as

327 demonstrated by the hPASK PAS-A and RE137 cases. In both cases, the $\operatorname{stdev}\left(\mathrm{c}_{i}\left[{ }^{15} \mathrm{~N}\right]\right)$

328 values of the apo proteins exceed the value of approximately $50\left(2 \times 10^{-10} \mathrm{ppm} / \mathrm{bar}^{2}\right)$

329 value we suggest as indicating potential ligand binding, and in both cases, we were able

330 to quickly identify compounds which bound to these proteins with micromolar affinities

331 from small libraries using either protein- or ligand-detected NMR methods. Our high 
332 pressure NMR results quickly add important information, showing that these compounds

333 bind in such a way as to reduce the flexibility of the receptors, probably through binding

334 internal cavities.

335 Finally, while our demonstrations have focused on relatively small $\sim 15 \mathrm{kDa}$ ligand

336 binding domains, we note that several routes can expand the reach of this approach to

337 larger and more complex systems by reducing spectral complexity. Options include

338 biochemical approaches to generate proteins with simplified ${ }^{15} \mathrm{~N}$-labeling patterns, as

339 provided by amino-acid type selective or sortase/intein-based domain-specific

340 techniques, to ${ }^{1} \mathrm{H} /{ }^{15} \mathrm{~N}$ TROSY or HNCO-type pulse sequences which can provide

341 improved resolution. Additional options may be provided by the improved NMR signal

342 properties of ${ }^{1} \mathrm{H} /{ }^{13} \mathrm{C}$ methyl signals for similar pressure-sensitive studies, particularly

343 given the placement of these residues near internal cavities.

\section{Materials and Methods}

Proteins were expressed in $E$. coli with uniform ${ }^{15} \mathrm{~N}$ labeling, purified using

combination of $\mathrm{Ni}(\mathrm{II})$ affinity and gel filtration chromatography before being exchanged

348 into a barostatic Tris:phosphate buffer mix which limits $\mathrm{pH}$ changes during

349 pressurization (52) and concentrated to $100-700 \mu \mathrm{M}$ for NMR spectroscopy. ${ }^{1} \mathrm{H} /{ }^{15} \mathrm{~N}$

$350 \mathrm{HSQC}$ spectra were acquired at increasing pressures from 20-2500 bar, interleaving

351 each high pressure spectrum with a low pressure (20 bar) spectrum to confirm that

352 changes in peak locations and intensities were reversible. All NMR data were

353 processed with NMRFx (One Moon Scientific) $(53,54)$ and analyzed with NMRViewJ

354 (One Moon Scientific) $(53,55)$. After individually processing each spectrum, we picked 
355 peaks and tracked their changes in chemical shifts as a function of pressure.

356 Separately handling movement in the ${ }^{1} \mathrm{H}$ and ${ }^{15} \mathrm{~N}$ dimensions, we fit these trends to a

357 second-order polynomial equation (Eq. 1, Fig. 2). Protein volume analyses of single

358 isolated cavities utilized cavfinder (1), while measurements of total void volumes utilized

359 ProteinVolume (5). Additional detailed procedures are found in SI Materials and

360 Methods.

361

\section{Acknowledgements}

$363 \quad$ The authors gratefully acknowledge useful discussions and software

364 contributions from Bruce Johnson (ASRC), assistance in sample preparation from Dong

365 Lee and Andrew Palacios (ASRC), and comments on manuscript by Xingjian Xu

366 (ASRC) and Hua Li (Chinese Academy of Sciences, Shanghai). U- ${ }^{15} \mathrm{~N}$ samples of

367 FABP proteins were generously provided by May Poh Lai and Ruth Stark (CCNY);

368 plasmids of Brd proteins were generously provided by Elena Rusinova and Ming-Ming

369 Zhou (Mt. Sinai Medical Center) while plasmids of CA01 were generously shared by

370 T.M. Jacobs and Brian KuhIman (UNC Chapel Hill). This work was supported by NSF

371 grant MCB-1818148 (K.H.G.) and NIH grant R01 GM106239 (K.H.G.), as well as a

372 postdoctoral fellowship to D.G. (number B3X from the Fonds de Recherche Québec -

373 Nature et Technologie (FRQNT)). 


\section{References}

376 1. T. H. Scheuermann et al., Allosteric inhibition of hypoxia inducible factor-2 with

2. T. H. Scheuermann et al., Isoform-Selective and Stereoselective Inhibition of Hypoxia Inducible Factor-2. J Med Chem 58, 5930-5941 (2015).

3. Y. Guo et al., Regulating the ARNT/TACC3 axis: multiple approaches to manipulating protein/protein interactions with small molecules. ACS Chem Bio/ 8, 626-635 (2013).

4. C. A. Amezcua, S. M. Harper, J. Rutter, K. H. Gardner, Structure and interactions of PAS kinase N-terminal PAS domain: model for intramolecular kinase regulation. Structure 10, 1349-1361 (2002).

5. C. R. Chen, G. I. Makhatadze, ProteinVolume: calculating molecular van der Waals and void volumes in proteins. BMC Bioinformatics 16, 101 (2015).

6. T. H. Scheuermann et al., Artificial ligand binding within the HIF2alpha PAS-B domain of the HIF2 transcription factor. Proc Natl Acad Sci U S A 106, 450-455 (2009).

7. J. Ashkani, D. J. Rees, The Critical Role Of VP1 In Forming The Necessary Cavities For Receptor-mediated Entry Of FMDV To The Host Cell. Sci Rep 6, 27140 (2016).

8. J. Mondal, N. Ahalawat, S. Pandit, L. E. Kay, P. Vallurupalli, Atomic resolution mechanism of ligand binding to a solvent inaccessible cavity in T4 lysozyme. PLoS Comput Biol 14, e1006180 (2018).

9. A. S. Tanwar, V. D. Goyal, D. Choudhary, S. Panjikar, R. Anand, Importance of Hydrophobic Cavities in Allosteric Regulation of Formylglycinamide Synthetase: Insight from Xenon Trapping and Statistical Coupling Analysis. PLOS ONE 8, e77781 (2013).

10. C. D. Andersson, B. Y. Chen, A. Linusson, Mapping of ligand-binding cavities in proteins. Proteins: Structure, Function, and Bioinformatics 78, 1408-1422 (2010).

11. Z. Guo et al., Identification of Protein-Ligand Binding Sites by the Level-Set Variational Implicit-Solvent Approach. Journal of Chemical Theory and Computation 11, 753-765 (2015).

12. Z. Zhang, Y. Li, B. Lin, M. Schroeder, B. Huang, Identification of cavities on protein surface using multiple computational approaches for drug binding site prediction. Bioinformatics 27, 2083-2088 (2011).

13. J. Dundas et al., CASTp: computed atlas of surface topography of proteins with structural and topographical mapping of functionally annotated residues. Nucleic Acids Res 34, W116-W118 (2006).

14. J.-K. Kim et al., BetaVoid: Molecular voids via beta-complexes and Voronoi diagrams: BetaVoid: Molecular Voids. Proteins: Structure, Function, and Bioinformatics 82, 1829-1849 (2014). 
15. K. P. Tan, T. B. Nguyen, S. Patel, R. Varadarajan, M. S. Madhusudhan, Depth: a web server to compute depth, cavity sizes, detect potential small-molecule ligandbinding cavities and predict the pKa of ionizable residues in proteins. Nucleic Acids Res 41, W314-W321 (2013).

16. Y. $\mathrm{Xu}$ et al., CavityPlus: a web server for protein cavity detection with pharmacophore modelling, allosteric site identification and covalent ligand binding ability prediction. Nucleic Acids Res 46, W374-W379 (2018).

17. D. A. Keedy et al., An expanded allosteric network in PTP1B by multitemperature crystallography, fragment screening, and covalent tethering. Elife 7, e36307 (2018).

18. C. Mattos, D. Ringe, Locating and characterizing binding sites on proteins. Nat Biotechnol 14, 595-599 (1996).

19. M. Gerstein, C. Chothia, Packing at the protein-water interface. Proc Natl Acad Sci U S A 93, 10167-10172 (1996).

20. K. Akasaka, Exploring the entire conformational space of proteins by high-pressure NMR. Pure and Applied Chemistry 75, 927-936 (2003).

21. K. Akasaka, Probing conformational fluctuation of proteins by pressure perturbation. Chem Rev 106, 1814-1835 (2006).

22. J. A. Caro, A. J. Wand, Practical aspects of high-pressure NMR spectroscopy and its applications in protein biophysics and structural biology. Methods 148, 67-80 (2018).

23. J. Roche et al., Cavities determine the pressure unfolding of proteins. Proc Natl Acad Sci U S A 109, 6945-6950 (2012).

24. H. R. Kalbitzer et al., Intrinsic allosteric inhibition of signaling proteins by targeting rare interaction states detected by high-pressure NMR spectroscopy. Angew Chem Int Ed Engl 52, 14242-14246 (2013).

25. K. Akasaka, H. Li, Low-Lying Excited States of Proteins Revealed from Nonlinear Pressure Shifts in ${ }^{1} \mathrm{H}$ and ${ }^{15} \mathrm{~N}$ NMR. Biochemistry 40, 8665-8671 (2001).

26. M. Gross, R. Jaenicke, Proteins under pressure. The influence of high hydrostatic pressure on structure, function and assembly of proteins and protein complexes. Eur J Biochem 221, 617-630 (1994).

27. B. L. Taylor, I. B. Zhulin, PAS domains: internal sensors of oxygen, redox potential, and light. Microbiol Mol Biol Rev 63, 479-506 (1999).

448 28. J. T. Henry, S. Crosson, Ligand-binding PAS domains in a genomic, cellular, and structural context. Annu Rev Microbiol 65, 261-286 (2011).

29. A. Losi, K. H. Gardner, A. Moglich, Blue-Light Receptors for Optogenetics. Chem Rev 118, 10659-10709 (2018).

30. K. Akasaka et al., Pressure response of protein backbone structure. Pressureinduced amide ${ }^{15} \mathrm{~N}$ chemical shifts in BPTI. Protein Sci 8, 1946-1953 (1999). 
454

31. T. Knubovets, J. J. Osterhout, P. J. Connolly, A. M. Klibanov, Structure, thermostability, and conformational flexibility of hen egg-white lysozyme dissolved in glycerol. Proc Natl Acad Sci U S A 96, 1262-1267 (1999).

32. Y. O. Kamatari, L. J. Smith, C. M. Dobson, K. Akasaka, Cavity hydration as a gateway to unfolding: an NMR study of hen lysozyme at high pressure and low temperature. Biophys Chem 156, 24-30 (2011).

33. W. G. Kaelin, Jr., P. J. Ratcliffe, Oxygen sensing by metazoans: the central role of the HIF hydroxylase pathway. Mol Cell 30, 393-402 (2008).

34. D. Wu, N. Potluri, J. Lu, Y. Kim, F. Rastinejad, Structural integration in hypoxiainducible factors. Nature 524, 303-308 (2015).

35. D. Wu et al., Bidirectional modulation of HIF-2 activity through chemical ligands. Nat Chem Biol 15, 367-376 (2019).

36. W. Chen et al., Targeting renal cell carcinoma with a HIF-2 antagonist. Nature 539, 112-117 (2016).

37. H. Cho et al., On-target efficacy of a HIF-2alpha antagonist in preclinical kidney cancer models. Nature 539, 107-111 (2016).

38. E. M. Wallace et al., A Small-Molecule Antagonist of HIF2alpha Is Efficacious in Preclinical Models of Renal Cell Carcinoma. Cancer Res 76, 5491-5500 (2016).

39. J. Key, T. H. Scheuermann, P. C. Anderson, V. Daggett, K. H. Gardner, Principles of ligand binding within a completely buried cavity in HIF2alpha PAS-B. J Am Chem Soc 131, 17647-17654 (2009).

40. F. Corrêa, J. Key, B. Kuhlman, Kevin H. Gardner, Computational Repacking of HIF-2a Cavity Replaces Water-Based Stabilized Core. Structure 24, 1918-1927 (2016).

41. J. Rutter, C. H. Michnoff, S. M. Harper, K. H. Gardner, S. L. McKnight, PAS kinase: An evolutionarily conserved PAS domain-regulated serine/threonine kinase. Proceedings of the National Academy of Sciences 98, 8991-8996 (2001).

42. A. Möglich, R. A. Ayers, K. Moffat, Structure and Signaling Mechanism of PerARNT-Sim Domains. Structure 17, 1282-1294 (2009).

43. V. Gonzalez et al., The partitioned Rhizobium etli genome: Genetic and metabolic redundancy in seven interacting replicons. Proceedings of the National Academy of Sciences 103, 3834-3839 (2006).

44. F. Correa, W. H. Ko, V. Ocasio, R. A. Bogomolni, K. H. Gardner, Blue light regulated two-component systems: enzymatic and functional analyses of lightoxygen-voltage (LOV)-histidine kinases and downstream response regulators. Biochemistry 52, 4656-4666 (2013).

45. G. Rivera-Cancel, W. H. Ko, D. R. Tomchick, F. Correa, K. H. Gardner, Full-length structure of a monomeric histidine kinase reveals basis for sensory regulation. Proc Natl Acad Sci U S A 111, 17839-17844 (2014). 
46. A. Vulpetti, U. Hommel, G. Landrum, R. Lewis, C. Dalvit, Design and NMR-Based Screening of LEF, a Library of Chemical Fragments with Different Local Environment of Fluorine. J Am Chem Soc 131, 12949-12959 (2009).

47. T. M. Jacobs et al., Design of structurally distinct proteins using strategies inspired by evolution. Science 352, 687-690 (2016).

48. J. Feng et al., A general strategy to construct small molecule biosensors in eukaryotes. Elife 4, e10606 (2015).

49. D. Sitkoff, D. A. Case, Theories of chemical shift anisotropies in proteins and nucleic acids. Progress in Nuclear Magnetic Resonance Spectroscopy 32, 165190 (1998).

50. M. Beck Erlach et al., Pressure dependence of side chain $13 \mathrm{C}$ chemical shifts in model peptides Ac-Gly-Gly-Xxx-Ala-NH2. J Biomol NMR 69, 53-67 (2017).

51. R. Kitahara, K. Hata, H. Li, M. P. Williamson, K. Akasaka, Pressure-induced chemical shifts as probes for conformational fluctuations in proteins. Progress in Nuclear Magnetic Resonance Spectroscopy 71, 35-58 (2013).

52. R. J. Quinlan, G. D. Reinhart, Baroresistant buffer mixtures for biochemical analyses. Anal Biochem 341, 69-76 (2005).

53. B. A. Johnson, From Raw Data to Protein Backbone Chemical Shifts Using NMRFx Processing and NMRViewJ Analysis. Methods Mol Biol 1688, 257-310 (2018).

54. M. Norris, B. Fetler, J. Marchant, B. A. Johnson, NMRFx Processor: a crossplatform NMR data processing program. J Biomol NMR 65, 205-216 (2016).

55. B. A. Johnson, R. A. Blevins, NMRView: a computer program for the visualization and analysis of NMR data. J Biomol NMR 4, 603-614 (1994). 